\title{
Occurrence of PER-1 Producing Clinical Isolates of Pseudomonas aeruginosa in Japan and their Susceptibility to Doripenem
}

\author{
Yoshinori Yamano, Toru Nishikawa, Takaji Fujimura, Takashi Yutsudou, \\ Masakatsu Tsuji, Hideaki Miwa
}

Received: July 13, 2006 / Accepted: November 20, 2006

(C) Japan Antibiotics Research Association

\begin{abstract}
The acquisition of resistance by extendedspectrum $\beta$-lactamases (ESBL) has been reported primarily for Enterobacteriaceae, but there are few reports on the isolation of ESBL-producing Pseudomonas aeruginosa. PER-1-type ESBL producing $P$. aeruginosa has been found in various regions around the world but there are no reports of clinical isolates in Japan. During our susceptibility surveillance studies over a 10 year period, we found four clinical isolates resistant to ceftazidime due to production of PER-1. They were resistant to ceftazidime but susceptible in the presence of clavulanic acid, a class A $\beta$-lactamase inhibitor. The strains had the ability to hydrolyze ceftazidime. They also had the gene for PER-1type ESBL. This is the first report of the isolation of PER-1 producing strains in Japan. These four strains were resistant to ceftazidime, cefepime and aztreonam with MICs of $64 \mu \mathrm{g} / \mathrm{ml}$ or more, but were more susceptible to carbapenem antibiotics. In particular, doripenem, which is a novel carbapenem antibiotic, showed good antibacterial activity with a MIC of 2 or $4 \mu \mathrm{g} / \mathrm{ml}$, which was more potent than meropenem and imipenem. Doripenem also showed good therapeutic efficacy against a systemic infection of mice with a PER-1 producing strain, and was also more potent in vivo than imipenem or meropenem.
\end{abstract}

Y. Yamano (Corresponding author), T. Nishikawa, T. Fujimura, T. Yutsudou, M. Tsuji, H. Miwa: Discovery Research Laboratories, Shionogi \& Co., Ltd., 3-1-1, Futaba-cho, Toyonaka, Osaka, 561-0825, Japan,

E-mail: yoshinori.yamano@shionogi.co.jp
Keywords Pseudomonas aeruginosa, doripenem, ESBL, PER-1, in vivo activity

\section{Introduction}

Pseudomonas aeruginosa is an opportunistic pathogen causing serious infection in immunocompromised hosts. $P$. aeruginosa has intrinsic resistance to many antibiotics and produces a variety of virulence factors. The intrinsic resistance of $P$. aeruginosa to various antibiotics is generally due to its low outer membrane permeability and the production of efflux pumps [1]. Although there are various antipseudomonal agents such as $\beta$-lactams, quinolones, and aminoglycosides, resistance to these antibiotics is acquired by $P$. aeruginosa at a relatively high rate by various resistant mechanisms.

P. aeruginosa, like other Gram-negative pathogens, is known to acquire resistance by producing various $\beta$ lactamases, which can be classified into chromosomal AmpC $\beta$-lactamase and plasmid-mediated $\beta$-lactamases. Among plasmid-mediated $\beta$-lactamases, extended-spectrum $\beta$-lactamases (ESBL) are generally known to hydrolyze cephamycins and/or carbapenems which can not be easily hydrolyzed by traditional $\beta$-lactamases [2]. Hence, ESBL production caused high resistance to various $\beta$-lactam antibiotics, which is a serious problem in clinic. Although the production of ESBL has been reported primarily in Enterobacteriaceae, there are few reports on the isolation of ESBL-producing $P$. aeruginosa [3]. PER-1-type $\beta$ lactamase was first detected as an ESBL in $P$. aeruginosa and was reported to hydrolyze various $\beta$-lactams except for 
imipenem, oxacillin and cephamycins $[4,5]$. Although there had been no reports on clinical isolates in Japan, PER-1 producing highly resistant strains have been found to be prevalent in P. aeruginosa and Acinetobacter spp. worldwide [6 12].

Among the antipseudomonal $\beta$-lactams, carbapenem antibiotics such as imipenem and meropenem display potent antipseudomonal activity and high stability to many of the serine-type $\beta$-lactamases including class C AmpC $\beta$ lactamase and ESBL. Doripenem, a novel carbapenem, has broad antibacterial spectrum and potent antipseudomonal activity [13 16]. In particular, the antibacterial activity of doripenem against clinical isolates of $P$. aeruginosa has been reported to be more potent than other carbapenem antibiotics [14 17]. In addition, doripenem and other carbapenem antibiotics have potent activity against ESBLproducing Enterobactriaceae [18].

In this study, we report the first isolation of PER-1 producing clinical isolates of $P$. aeruginosa in Japan, and the in vitro and in vivo antibacterial activities of doripenem against PER-1 producing $P$. aeruginosa.

\section{Materials and Methods}

\section{Bacterial Strains and Susceptibility Testing}

P. aeruginosa SR24836, SR24837, SR24838, SR24839 and SR24840 were isolated at a Japanese clinical facility in the year 2002. P. aeruginosa SR24823, SR24897, SR24858, and SR27001 were isolated at other Japanese clinical facilities in 2002. P. aeruginosa PAO1 and SR24 are generally susceptible to antipseudomonal antibiotics, and strain SR24-12, which is a resistant mutant derived from strain SR24, displays constitutive production of chromosomal AmpC $\beta$-lactamase.

\section{Antibiotics}

Doripenem was synthesized at Shionogi \& Co., Ltd. Meropenem, imipenem, ceftazidime, cefepime and aztreonam were purchased from U.S. Pharmacopeia (Rockville, MD). Clavulanic acid, a $\beta$-lactamase inhibitor, and cilastatin, a dehydropeptidase-I inhibitor, were also purchased from U.S. Pharmacopeia.

\section{Susceptibility Testing}

MICs were determined using cation-adjusted MuellerHinton broth (Becton Dickinson, Sparks, MD) by the microbrothdilution method according to the Clinical and Laboratory Standards Institution guidelines (formerly National Committee for Clinical Laboratory Standards) [19]. For the detection of ESBL production, MICs were determined in the presence of clavulanic acid, an inhibitor for the class A $\beta$-lactamases.

\section{Detection of ESBL Genes}

The genes for five types of ESBL (PER, VEB, GES, SHV and TEM-types) were detected by polymerase chain reaction (PCR), which was performed using the primers described by Weldhagen et al. [3]. The complete PER-1 gene was amplified by using two primers, which correspond to $N$-terminal or $C$-terminal regions, followed by determination of the nucleotide sequence by the direct sequencing method.

\section{Preparation of $\beta$-Lactamase}

A crude cell extract of SR24840 was prepared by disruption of exponentially growing cells by repeated freeze-thawing and sonication at $4{ }^{\circ} \mathrm{C}$, followed by removal of the cell debris by centrifugation at $8,000 \times g$ for 15 minutes. The supernatant, as a crude cell extract, was prepared by centrifugation at $15,000 \times g$ for 30 minutes, followed by filtration through a filter with a pore size of $0.22 \mu \mathrm{m}$. The protein content of the crude cell extract was determined using a BCA protein assay kit (Pierce, Rockford, IL). The hydrolysis of ceftazidime and nitrocefin was examined by incubation of $100 \mu \mathrm{M}$ ceftazidime and nitrocefin at $30^{\circ} \mathrm{C}$ in the presence of diluted crude cell extracts. The hydrolysis rates of ceftazidime and nitrocefin were determined by photometric methods at wavelengths of 260 and $490 \mathrm{~nm}$, respectively [20, 21].

\section{Analysis of Macrorestriction Patterns of Genomic DNA}

The analysis was performed using genomic DNA of PER-1 producing clinical isolates by the pulsed-field gel electrophoresis (PFGE) reported by Pagani et al. [11]. Briefly, genomic DNA was extracted from the clinical isolates and was digested with SpeI using CHEF Genomic DNA Plug Kits (Bio-rad, Hercules, CA). The digested DNA fragments were separated on agarose gel by PFGE using the CHEF-DRII system (Bio-rad).

\section{Therapeutic Efficacy against Systemic Infection Model}

Five-week-old female mice were infected intraperitoneally with $0.5 \mathrm{ml}$ of the bacterial suspension of strain SR24840 containing $6.4 \times 10^{5} \mathrm{cfu} / \mathrm{ml}$. All test antibiotics were subcutaneously administered at 1,3 and 5 hours after infection. For the carbapenem antibiotics doripenem, meropenem and imipenem, equal amounts of cilastatin was simultaneously administered to prevent the degradation of carbapenem antibiotics by renal dehydropeptidase-I [22, 23]. Seven mice were used at each dosage of the compound. The efficacy was determined from the survival 
Table 1 Susceptibility of ceftazidime-resistant strains of Pseudomonas aeruginosa to various $\beta$-lactam antibiotics

\begin{tabular}{|c|c|c|c|c|c|c|}
\hline & Ceftazidime & Cefepime & Doripenem & Meropenem & Imipenem & Aztreonam \\
\hline SR24837 & $>64$ & $>64$ & 4 & 8 & 16 & $>64$ \\
\hline SR24838 & $>64$ & 64 & 2 & 4 & 16 & $>64$ \\
\hline SR24839 & $>64$ & $>64$ & 4 & 8 & 16 & $>64$ \\
\hline SR24840 & $>64$ & 64 & 2 & 8 & 16 & $>64$ \\
\hline SR24 & 1 & 0.5 & 0.125 & 0.25 & 1 & 4 \\
\hline SR24-12 & 64 & 64 & 0.25 & 0.25 & 1 & $>64$ \\
\hline
\end{tabular}

MICs $(\mu \mathrm{g} / \mathrm{ml})$ were determined by microdilution method using cation-adjusted Mueller-Hinton medium.

Table 2 Effect of clavulanic acid on susceptibility of the strains to ceftazidime

\begin{tabular}{lrr}
\hline & \multicolumn{2}{c}{ MIC of ceftazidime in the presence of } \\
\cline { 2 - 3 } & None & $10 \mu \mathrm{g} / \mathrm{ml}$ of clavulanic acid \\
\hline SR24837 & $>64$ & 2 \\
SR24838 & $>64$ & 2 \\
SR24839 & $>64$ & 2 \\
SR24840 & $>64$ & 2 \\
SR24 & 1 & 1 \\
SR24-12 & 64 & 64 \\
\hline
\end{tabular}

MICs $(\mu \mathrm{g} / \mathrm{ml})$ were determined by microdilution method using cationadjusted Mueller-Hinton medium.

rates of the mice at 7 days after infection, and $\mathrm{ED}_{50}$ and $95 \%$ confidence limits were calculated by the logit method.

\section{Results}

Occurrence of Ceftazidime-resistant Strains and Their Susceptibilities to Various Antibacterials

We have been performing susceptibility surveillance studies every two years since 1992 using clinical isolates collected from about 17 medical facilities in Japan [24 29]. For P. aeruginosa, about 100 strains have been collected each year. We found that four strains isolated at one medical facility in 2002 had unique characteristics. The strains were highly resistant to ceftazidime with MIC of $>64 \mu \mathrm{g} / \mathrm{ml}$ (Table 1), but were susceptible to ceftazidime in the presence of $10 \mu \mathrm{g} / \mathrm{ml}$ of clavulanic acid, an inhibitor for class A $\beta$-lactamase (Table 2). Other ceftazidime-resistant strains, which were isolated at a frequency of about 10 to $20 \%$ in each surveillance study, were resistant even in the presence of clavulanic acid, suggesting that they did not

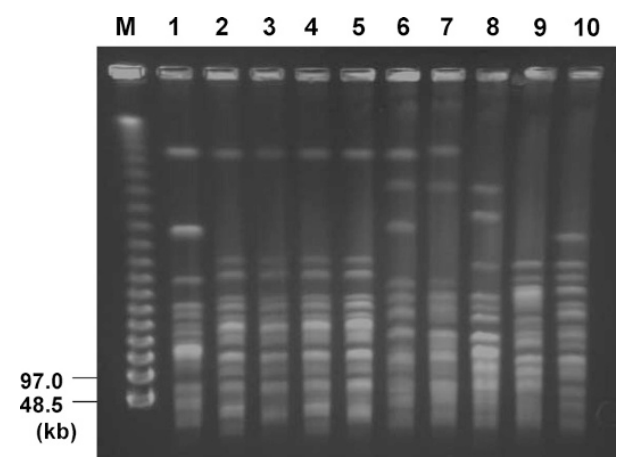

Fig. 1 Pulse field gel electrophoresis profiles of clinical isolates.

Spel-digested DNA fragments of genomic DNA derived from PER-1 producing strains SR24837 (lane 2), SR24838 (lane 3), SR24839 (lane 4) and SR24840 (lane 5), and non PER-1 producing strains SR24836 (lane 1), SR24823 (lane 6), SR24897 (lane 7), SR24858 (lane 8) and SR27001 (lane 9) were separated on agarose gel by pulse field gel electrophoresis apparatus. Lambda ladder markers were shown on lane $\mathrm{M}$.

produce class A $\beta$-lactamase including ESBL like PER-1. Generally, many of the ceftazidime-resistant strains as well as strain SR24-12 were reported to produce constitutively chromosomal AmpC $\beta$-lactamase, and were not susceptible to ceftazidime even in the presence of clavulanic acid. Some strains have been reported to acquire high resistance to ceftazidime even in the presence of clavulanic acid due to the production of class B metallo- $\beta$-lactamase.

Because these four resistant strains were isolated at one medical facility, the isogenicity between these four strains was observed by comparing the macro restriction patterns of genomic DNA of these strains by PFGE. Figure 1 showed that these four strains showed almost identical macro restriction patterns, although other strains showed significantly different macro restriction patterns. These results suggested that these four strains were isogenic, so strain SR24840 was used for further studies. 
Table 3 Hydrolysis of $\beta$-lactams by PER-1 producing strains

\begin{tabular}{ccc}
\hline & Hydrolysis rate $(\mathrm{mM} / \mathrm{min} / \mathrm{mg}$-protein) \\
\cline { 2 - 3 } & Nitrocefin & Ceftazidime \\
\hline SR24840 & 280 & 2.1 \\
SR24-12 & 1160 & $<0.01$ \\
\hline
\end{tabular}

The hydrolysis rate was determined by a photometric method at the concentration of $100 \mu \mathrm{M}$ substrate.

The hydrolysis of ceftazidime by the cell free extracts of strain SR24840 was detected, but not by the cell free extract of SR24-12, AmpC producer (Table 3). On the other hand, the hydrolysis of nitrocefin did not differ significantly between these two strains. These results strongly suggested that strain SR24840 had the ability to hydrolyze ceftazidime.

\section{Detection of PER-1 Production}

There have been few reports on the production of ESBL in P. aeruginosa, but some ESBLs are known to cause high resistance in P. aeruginosa [3]. The presence of genes for various ESBLs having the ability to hydrolyze ceftazidime was investigated using PCR. As a result, the PER-1 gene was detected in all four strains, and the nucleotide sequence of the amplified PER-1 gene of SR24840 was identical to that of the PER-1 gene which was previously reported [5].

These results showed that these four strains were resistant to ceftazidime because they were PER-1 producers. This is the first report of the isolation of PER-1 producing strains of $P$. aeruginosa in Japan.

\section{Therapeutic Efficacy against Systemic Infection Models}

As shown in Table 1, these strains were highly resistant to ceftazidime, cefepime and aztreonam with MIC of $64 \mu \mathrm{g} / \mathrm{ml}$ or more. On the other hand, they were more susceptible to carbapenem antibiotics, with doripenem showing the most potent activity with MIC of 2 to $4 \mu \mathrm{g} / \mathrm{ml}$. The MIC of meropenem against these four strains was 2- to 4-fold higher than that of doripenem, and the MIC of imipenem was 4- to 8-fold higher than that of doripenem.

The efficacy of various $\beta$-lactams was tested in mice infected with PER-1 producing strain SR28480. For carbapenem antibiotics, cilastatin, an inhibitor of DHP-I, was administered simultaneously at a ratio of $1: 1$ in order to inhibit the effect of murine dehydropeptidase-I on pharmacokinetic profiles. Ceftazidime and cefepime were not active in this model, however, carbapenem antibiotics
Table 4 Therapeutic efficacy against murine systemic infection caused by $P$. aeruginosa

\begin{tabular}{lcl}
\hline & $\mathrm{ED}_{50}(\mathrm{mg} / \mathrm{kg})$ & $95 \%$ confidence limit \\
\hline Doripenem & 2.83 & $1.37 \sim 6.42$ \\
Meropenem & 7.72 & $3.55 \sim 15.7$ \\
Imipenem & 5.52 & $2.72 \sim 12.3$ \\
Ceftazidime & $>32$ & $\mathrm{NC}$ \\
Cefepime & $>32$ & $\mathrm{NC}$ \\
\hline
\end{tabular}

Infection: Five-week-old female mice were infected intraperitoneally with $6.4 \times 10^{5} \mathrm{cfu} / \mathrm{ml}$ of $P$. aeruginosa SR24840. Treatment: Antibiotics were subcutaneously administered at 1,3 and 5 hours after infection. Doripenem, meropenem and imipenem were administered in concentrations equal to cilastatin. NC: not calculated.

showed good therapeutic efficacy. The therapeutic efficacy of doripenem was 2- and 3-fold better than that of imipenem and meropenem (Table 4). These results showed that doripenem was also the most effective even against infection models caused by a PER-1 producing strain of $P$. aeruginosa.

\section{Discussion}

We report the first instance of isolation of PER-1 producing strains of $P$. aeruginosa in Japan. In 1991, PER-1 producing strains of $P$. aeruginosa were found in Turkey [4] and have been reported in European countries and USA [8 12]. However, there had been no reports on the emergence of PER-1 producing strains of P. aeruginosa in Asian countries, although some strains of Acinetobacter spp. were reported to carry PER-1 in various countries including Korea [30]. PER-1 was reported to be transferable to various Gram-negative bacteria [3], and strains carrying PER- 1 and metallo- $\beta$-lactamase or OXAtype ESBL have also been isolated [12,31].

In the case of the four strains discovered in this study, the PFGE and antibiotic susceptibility profiles were almost identical although they were different from those of other strains without PER-1 production (Fig. 1). These results strongly suggest the spread of an isogenic resistant strain in a hospital. Although no other PER-1 producing $P$. aeruginosa has yet been reported in Japan, carefully monitoring is needed.

Doripenem is a novel carbapenem which has a broad antibacterial spectrum and potent antipseudomonal activity. In particular, the activity of doripenem against clinical isolates of $P$. aeruginosa was reported to be superior to 
other carbapenem antibiotics [14 17]. Doripenem was also reported to be active against PER-1 producing transconjugants susceptible to ceftazidime and cefepime [32]. This study showed that doripenem was active against PER-1 producing clinical isolates which were resistant to ceftazidime and cefepime. The differences of susceptibility between PER-1 producers might be due to the amount of production of PER-1.

Doripenem and meropenem can be used clinically without a dehydropeptidase-I inhibitor due to their high stability to human renal dehydropeptidase-I [22, 33]. Doripenem is also more stable against murine dehydropeptidase-I than meropenem and imipenem, and the therapeutic efficacy by single administration of doripenem against various infection models has been found to be comparable to or better than that of the combinational administration of meropenem: cilastatin and imipenem : cilastatin $[14,16]$. However, Tsuji et al. also reported that the pharmacokinetic profile of doripenem was improved by cilastatin and suggested evaluation by the combination administration of doripenem and cilastatin [14]. Hence, the therapeutic efficacy of carbapenem antibiotics including doripenem was evaluated by combination use with cilastatin in this study. Under these conditions, doripenem was shown to have potent therapeutic efficacy against $P$. aeruginosa which produces PER-1 ESBL, being more potent than meropenem and imipenem.

These results showed that, among carbapenem antibiotics tested, doripenem displays the most potent therapeutic efficacy against $P$. aeruginosa, regardless of PER-1 production.

\section{References}

1. Li XZ, Livermore DM, Nikaido H. Role of efflux pumps in intrinsic resistance of Pseudomonas aeruginosa: active efflux as a contributing factor to $\beta$-lactam resistance. Antimicrob Agents Chemother 38: 1742-1752 (1994)

2. Jarlier V, Nicolas MH, Fournier G, Phillippon A. Extended broad-spectrum $\beta$-lactamases conferring transferable resistance to newer $\beta$-lactam agents in Enterobacteriaceae: hospital prevalence and susceptibility patterns. Rev Infect Dis 10: 867-878 (1988)

3. Weldhagen GF, Poirel L, Nordmann P. Amber class A extended-spectrum $\beta$-lactamases in Pseudomonas aeruginosa: novel developments and clinical impact. Antimicrob Agents Chemother 47: 2385-2392 (2003)

4. Nordmann P, Ronco E, Naas T, Duport C, Michel-Briand Y, Labia R. Characterization of a novel extended-spectrum $\beta$ lactamase from Pseudomonas aeruginosa. Antimicrob
Agents Chemother 37: 962-969 (1993)

5. Nordmann P, Naas T. Sequence analysis of PER-1 extendedspectrum $\beta$-lactamase from Pseudomonas aeruginosa and comparison with class A $\beta$-lactamases. Antimicrob Agents Chemother 38: 104-114 (1994)

6. Vahaboglu H, Coskunkan F, Tansel O, Ozturk R, Sahin N, Koksal I, Kocazeybek B, Tatman-Otkun M, Leblebicioglu H, Ozinel MA, Akalin H, Kocagoz S, Korten V. Clinical importance of extended-spectrum $\beta$-lactamase (PER-1type)-producing Acinetobacter spp. and Pseudomonas aeruginosa strains. J Med Microbiol 50: 642-645 (2001)

7. Kolayli F, Gacar G, Karadenizli A, Sanic A, Vahaboglu H. PER-1 is still widespread in Turkish hospitals among Pseudomonas aeruginosa and Acinetobacter spp. FEMS Microbiol Lett 249: 241-245 (2005)

8. Luzzaro F, Mantengoli E, Perilli M, Lombardi G, Orlandi V, Orsatti A, Amicosante G, Rossolini GM, Toniolo A. Dynamics of a nosocomial outbreak of multidrug-resistant Pseudomonas aeruginosa producing the PER-1 extendedspectrum $\beta$-lactamase. J Clin Microbiol 39: 1865-1870 (2001)

9. Claeys G, Verschraegen G, De Baare T, Vaneechoute M. PER-1 $\beta$-lactamase-producing Pseudomonas aeruginosa in an intensive care unit. J Antimicrob Chemother 45: 924-925 (2000)

10. De Champs C, Chanal C, Sirot D, Baraduc R, Romaszko JP, Bonnet R, Plaidy A, Boyer M, Carroy E, Gbadamassi MC, Laluque S, Oules O, Poupart MC, Villemain M, Sirot J. Frequency and diversity of class A extended-spectrum $\beta$ lactamase in hospital of the Auvergne, France: a 2 year prospective study. J Antimicrob Chemother 54: 634-639 (2004)

11. Pagani L, Mantengoli E, Migliavacca R, Nucleo E, Pollini S, Spalla M, Daturi R, Romero E, Rossolini GM. Multifocal detection of multidrug-resistant Pseudomonas aeruginosa producing the PER-1 extended-spectrum $\beta$-lactamase in Northern Italy. J Clin Microbiol 42: 2523-2529 (2004)

12. Docquier JD, Luzzaro F, Amicosante G, Toniolo A, Rossolini GM. Multidrug-resistant Pseudomonas aeruginosa producing PER-1 extended-spectrum serine- $\beta$ lactamase and VIM-2 metallo- $\beta$-lactamase. Emerg Infect Dis 7: 910-911 (2001)

13. Iso $\mathrm{Y}$, Irie $\mathrm{T}$, Iwaki $\mathrm{T}$, Kii $\mathrm{M}$, Sendo $\mathrm{Y}$, Motokawa $\mathrm{K}$, Nishitani Y. Synthesis and modification of a novel 1- $\beta$ methyl carbapenem antibiotic, S-4661. J Antibiot 49: 478-484 (1996)

14. Tsuji M, Ishii Y, Ohno A, Miyazaki S, Yamaguchi K. In vitro and in vivo antibacterial activities of S-4661, a new carbapenem. Antimicrob Agents Chemother 42: 94-99 (1998)

15. Fujimura T, Kimura Y, Yoshida I, Higashiyama I, Jinushi Y, Munekage T, Kuroda N, Doi M, Nishikawa T, Yamano Y. In vitro antibacterial activity of doripenem, a novel parenteral carbapenem. Jpn J Chemother 53 (S-1): 57-70 (2005)

16. Miwa H, Kimura Y, Jinushi Y, Fujimura T, Nishikawa T, 
Munekage T, Kuroda N, Yamano Y, Tsuji M, Okazaki K, Sato T, Matsuda H. Antipseudomonal activity of doripenem, a novel parenteral carbapenem. Jpn J Chemother 53 (S-1): 80-91 (2005)

17. Ge Y, Wikler MA, Sahm DF, Blosser-Middleton RS, Karlowsky JA. In vitro antimicrobial activity of doripenem, a new carbapenem. Antimicrob Agents Chemother 48: 1384-1396 (2004)

18. Mushtaq S, Ge Y, Livermore DM. Comparative activities of doripenem versus isolates, mutants, and transconjugants of Enterobacteriaceae and Acinetobacter spp. with characterized $\beta$-lactamases. Antimicrob Agents Chemother 84: 1313-1319 (2004)

19. National Committee for Clinical Laboratory Standards (NCCLS): Performance standards for antimicrobial susceptibility testing. Twelfth Informational Supplement M100-S12. Wayne, PA: NCCLS, 2002

20. Jason JAT. A direct spectrometric assay for penicillin $\beta$ lactamase (penicillinase). Biochim Biophys Acta 99: 171-172 (1965)

21. O'Callghan CH, Morris A, Kirby SM, Singler AH. Novel method for detection of $\beta$-lactamases by using a chromogenic cephalosporin substrate. Antimicrob Agents Chemother 1: 283-288 (1972)

22. Mouton JW, Touzw DJ, Horrevorts AM, Vinks AA. Comparative pharmacokinetics of the carbapenems: clinical implications. Clin Pharmacokinet 39: 185-201 (2000)

23. Sato T, Tsuji M, Okazaki K, Matsuda H, Yoshitomi T, Miwa H. Therapeutic efficacy of doripenem, a novel parenteral carbapenem antibiotic, against experimental infection in mice and rats. Jpn J Chemother 53 (S-1): 71-79 (2005)

24. Sasaki S, Nagano K, Kimura Y, Jinushi Y, Nagata H, Uotani K, Higashiyama H. Surveillance of the susceptibility of clinical isolates of various bacterial species to antibacterial agents. Jpn J Chemother 43: 12-26 (1995)

25. Nagano K, Kimura Y, Higashiyama I, Jinushi Y, Sasaki S, Yoshida I. Surveillance of susceptibility of clinical isolates of various bacterial species to antibacterial agents: No. 2 . against gram-negative bacteria in 1994. Jpn J Chemother 44:
610-625 (1996)

26. Yoshida I, Nagano K, Kimura Y, Higashiyama I, Sasaki S. Surveillance of susceptibility of clinical isolates of various bacterial species to antibacterial agents: No. 2. against gramnegative bacteria isolated in 1996. Jpn J Chemother 46: 343-362 (1998)

27. Yoshida I, Higashiyama I, Kimura Y, Sasaki S. Surveillance of susceptibility of clinical isolates of various bacterial species to antibacterial agents: No. 2. gram-negative bacteria isolated in 1998. Jpn J Chemother 48: 610-632 (2000)

28. Yoshida I, Sugimori G, Higashiyama I, Kimura Y, Yamano Y. Surveillance of susceptibility of clinical isolates of various bacterial species to antibacterial agents: antimicrobial activity against gram-negative bacteria isolated in 2000. Jpn J Chemother 51: 209-232 (2003)

29. Yoshida I, Fujimura T, Jinushi Y, Higashiyama I, Kimura Y, Sugimori G, Yamano Y. Surveillance of susceptibility of clinical isolates to antibacterial agents: antibacterial activity against aerobic gram-negative bacteria isolated in 2002. Jpn $\mathrm{J}$ Chemother, in press

30. Yong D, Shin JH, Kim S, Lim Y, Yum JH, Lee K, Chong Y, Bauernfeind Y. High prevalence of PER-1 extendedspectrum $\beta$-lactamase-producing Acinetobacter spp. In Korea. Antimicrob Agents Chemother 47: 1749-1751 (2003)

31. Aktas Z, Poirel L, Salcioglu M, Ozcan PE, Midilli K, Bal C, Ang O, Nordmann P. PER-1 and OXA-10-like $\beta$-lactamases in ceftazidime-resistant Pseudomonas aeruginosa isolates from intensive care unit patients in Istanbul, Turkey. Clin Microbiol Infect 11: 193-198 (2005)

32. Mushtaq S, Ge Y, Livermore DM. Doripenem versus Pseudomonas aeruginosa in vitro: activity against characterized isolates, mutants, and transconjugants and resistance selection potential. Antimicrob Agents Chemother 48: 3086-3092 (2004)

33. Yamano Y, Kawai Y, Yutsudo T. Stability of doripenem against human renal dehydropeptidase-I. Jpn J Chemother 53 (S-1): 92-95 (2005) 